\title{
Lagrangian mixing in an axisymmetric hurricane model
}

\author{
B. Rutherford ${ }^{1}$, G. Dangelmayr ${ }^{1}$, J. Persing ${ }^{1}$, M. Kirby ${ }^{1}$, and M. T. Montgomery ${ }^{2}$ \\ ${ }^{1}$ Department of Mathematics, Colorado State University, Fort Collins, CO 80523-1874, USA \\ ${ }^{2}$ Department of Meteorology, Naval Postgraduate School, Monterey, CA 93943-5114, USA
}

Received: 23 July 2009 - Published in Atmos. Chem. Phys. Discuss.: 9 September 2009

Revised: 15 June 2010 - Accepted: 15 July 2010 - Published: 23 July 2010

\begin{abstract}
This paper discusses the extension of established Lagrangian mixing measures to make them applicable to data extracted from a 2-D axisymmetric hurricane simulation. Because of the non-steady and unbounded characteristics of the simulation, the previous measures are extended to a moving frame approach to create time-dependent mixing rates that are dependent upon the initial time of particle integration, and are computed for nonlocal regions. The global measures of mixing derived from finite-time Lyapunov exponents, relative dispersion, and a measured mixing rate are applied to distinct regions representing different characteristic feautures within the model. It is shown that these time-dependent mixing rates exhibit correlations with maximal tangential winds during a quasi-steady state, establishing a connection between mixing and hurricane intensity.
\end{abstract}

\section{Introduction}

The question of the interaction between different characteristic regions of a hurricane, in particular the eye, eyewall, and near-core, is considered of fundamental importance in the study of structure and intensity, Frank and Ritchie (1999), Frank and Ritchie (2001), Kossin and Eastin (2001), Kossin and Schubert (2001), Schubert et al. (1999), Willoughby (2001). In particular, mixing in the lower troposphere at the eye-eyewall interface, Cram et al. (2007), Montgomery et al. (2006), Persing and Montgomery (2003), has been proposed to play an important role for intensification. The proposed mechanisms are either direct and mechanical or indirect and thermodynamic. Direct and mechanical mechanisms reduce intensity as air with low absolute angular momentum from the eye is stirred to the radius of maximum winds (RMW).

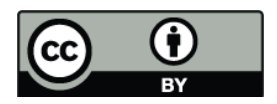

Correspondence to: B. Rutherford (rutherfo@math.colostate.edu)
Indirect and thermodynamic mechanisms stir air with high entropy from the eye to the eyewall that will generate enhanced local buoyancy, Smith et al. (2005), Braun (2002), Zhang and Yau (2002), leading to an enhanced energetic cycle for the hurricane as a whole (e.g. as a modified heat cycle). Maximum tangential winds (found in the eyewall generally at $z \approx 1 \mathrm{~km}$ ) will be used here as the principal measure of intensity.

Mixing in hurricanes is often viewed in an Eulerian manner, based on the instantaneous velocity fields. If the velocity fields are time varying, the Eulerian structures may not be representative of the actual particle motion. In recent work in fluid dynamics, time dependent invariant manifolds, i.e. curves on which particle trajectories remain, have been studied that partition the domain into distinct regions and are visualized as local maxima of Lagrangian stretching fields, Haller (2002), Haller and Poje (1997), Haller and Yuan (2000), Haller (2000), see Rutherford et al. (2010) for a recent application to a 2-D hurricane-like vortex model. Most of these studies are for time-varying 2-D velocity fields in closed and bounded domains. An extension of the use of these methods to the 3-D case is given by Green et al. (2006). In this paper we investigate a 2-D flow that is more complicated because the domain is unbounded and there is an inflow and outflow. Lagrangian structures associated with separation from boundaries have been identified by Haller (2004) and Surana and Haller (2008), and generally differ from the Eulerian separation points.

Statistical measures of Lagrangian mixing have been applied to 2-D fluid models by Voth et al. (2003) and Antonsen Jr. et al. (1996), but the mixing characteristics are time-dependent only in the sense that they vary with the integration time. While this is sufficient for steady or periodic velocity fields, in general time-varying velocity fields there is also a significant dependence on the initial time at which the trajectories are seeded. This holds for all statistical measures used so far, including relative dispersion, which has

Published by Copernicus Publications on behalf of the European Geosciences Union. 
been used to diagnose atmospheric mixing by Huber et al. (2001) in a limited way in a global circulation problem.

In this paper we apply Lagrangian techniques to study mixing in the axisymmetric hurricane model of Rotunno and Emanuel (1987). The model of the hurricane shows the principal structures of 3-D hurricanes (e.g., eye, eyewall updraft, near-surface inflow, and outflow jet), while resolving the $2 \mathrm{D}$ velocity fields in the radial and vertical directions. The advantages of axisymmetric models are that the size of the problem is reduced and the geometry is simpler. The structures found to be characteristic for the mixing processes within an axisymmetric model may, with caution, be extended to give clues about mixing within 3-D models or reality. The model of Rotunno and Emanuel (1987) yields time-dependent 2-D velocity fields that show complex spatial and temporal variation about a quasi-steady state, leading to a variety of dynamically interesting, time-dependent structures. The temporal complexity of the velocity fields makes the extraction of coherent structures difficult, as structures may have very short times of existence.

Given the complexity and time-dependent nature of the velocity field in the Rotunno and Emanuel model, it is neccessary to develop a hybrid (local-global) approach to measuring mixing rates. Local Eulerian flow structures are generally not valuable for characterizing mixing in the entire flow if the structures do not exist in a coherent manner. However, global measures of mixing are not suitable for this model either, because much of the mixing occurs around the eyewall updraft region, which is where the maximum winds occur. Outer environment and eye behavior are very separate processes from the mixing that occurs in and around the updraft, hence diagnosing the mixing for the entire domain from a single measure is not reasonable. Most current methods are either local or global.

Local methods established in the Lagrangian frame study particular features such as hyperbolic trajectories and their stable and unstable manifolds, and track the effects of these features. Global measures attempt to define a rate of mixing that is representative of the entire system. To diagnose mixing in a domain that has distinct mixing regions, which have little interaction with other regions, we adapt both local and global mixing diagnostics to quantify mixing between nonlocal regions. The nonlocality of the regions requires extracting mixing measures from ensembles of trajectories, which makes these measures statistical in nature.

Our approach to solving the hurricane mixing problem will be guided by considering time and space dependence of mixing processes. The dynamically distinct regions of hurricanes (e.g., the eye, the eyewall, near-core, etc.) require that the space dependence of mixing properties follows a regional approach. The domain is partitioned into regions, and a mixing rate is calculated for each region, giving a spatial dependence to the mixing rates. For general time dependence, not only variations in the integration time, but also variations in the initial time have to be used to define the mixing rates. The result is a time series of mixing rates computed for each spatial region. The initial time-dependent mixing rates are then compared with measures of intensity to establish correlations between these characteristic quantities. The correlation analysis shows that the mixing rates computed for some of the regions are sigificantly correlated with the maximum tangential winds.

A mixing rate is a measure of how quickly an initial tracer in a fluid becomes homogenized. The homogenization process has been studied for autonomous or time-periodic velocity fields in bounded and closed domains, and gives a mixing rate for the entire system. This rate can be compared to other rates derived from measures of advection or diffusion. Advective mixing concerns reversible transport dictated by the structure of flow boundaries, while irreversible diffusive mixing occurs through filamentation of the boundaries. The fast convergence in integration time of advective measures makes them more suitable for this model. Since the advective measures presented here follow from particle trajectories, the associated mixing rates are Lagrangian in nature, and measure the interaction of features that move with the flow.

The outline of the paper is as follows. Section 2 gives an overview of current Lagrangian mixing rates. In Sect. 3 we describe the characteristics of the axisymmetric model that is used for this study. The adaptation of current methods to make them applicable to our non-steady and open fluidflow problem, along with the numerical methods used is described in Sect. 4. The results of our study are presented in Sects. 5-8. In Sect. 5 we show and discuss the Lagrangian scalar fields. Section 6 gives a Lagrangian characterization of the eye-eyewall interaction, and Sect. 7 shows how the Lagrangian structures are related to low and high intensity steady state approximations. In Sect. 8, we analyze correlations between measures of intensity and mixing rates. A discussion and conclusions are given in Sect. 9.

\section{Overview of current Lagrangian methods}

Lagrangian mixing measures have advantages over Eulerian measures for their applicability to time dependent fluid flows. For time dependent flows, trajectories may cross Eulerian boundaries, and diverge from instantaneous features of the flow. Lagrangian techniques capture the total separation of trajectories and provide structures that are invariant under the flow. The local techniques quantify the local rate of stretching of an initial area element. The locations of highest stretching may be used to define invariant manifolds which give insight into the transport properties. These techniques are useful for incompressible flows where the velocity field varies slowly both in space and time. Some of the local techniques currently in use are finite-time Lyapunov exponents, (Haller, 2002; Haller and Poje, 1997; Haller and Yuan, 2000), and direct Lyapunov exponents (Haller, 2001). Distinguished hyperbolic trajectories have been studied by 
Haller (2001), Ide et al. (2002), and Salman et al. (2008). Finite-size Lyapunov exponents have been used by Artale et al. (1997), d'Ovidio et al. (2001), and Green et al. (2006), and applied in a study of Koh and Legras (2002) to the stratospheric polar vortex. Relative dispersion was studied by $\mathrm{Hu}-$ ber et al. (2001) to diagnose transport in the troposphere.

Global Lagrangian techniques provide representative mixing characteristics of an entire domain, without exact extraction of structures, and are statistical in nature. Global mixing measures have been applied to flows in bounded domains without a dominant jet, and with with no general time dependence. The global measures are related to the homogenization of a tracer within the domain, and are usually extensions of local measures to the entire domain. The measured mixing rate determines how fast the tracer is homogenized, (Voth et al., 2003). Another global mixing rate is defined through the distribution of the values of finite-time Lyapunov exponents, and is an extension of the local measure of advection to the entire domain, see Antonsen Jr. et al. (1996).

\subsection{Measured mixing rate (MMR)}

The mixing rate of a system can be measured by calculating the rate at which an initial tracer becomes homogenized by the flow. Let

$\mathbf{x}_{\mathbf{0}} \mapsto \phi_{t_{0}}^{t}\left(\mathbf{x}_{\mathbf{0}}\right)$

be the flow map from time $t_{0}$ to time $t$ associated with a 2$\mathrm{D}$ non-steady velocity field $\mathbf{v}(\mathbf{x}, t)$, that is, the solution of $\dot{\mathbf{x}}=\mathbf{v}(\mathbf{x}, t)$ with initial condition $\mathbf{x}\left(t_{0}\right)=\mathbf{x}_{0}$. If an initial tracer is planted uniformly over a subdomain at time $t_{0}$ and evolved, then the variance of the tracer concentration should decay over time as the tracer fills the entire domain. If $\rho_{0}(\mathbf{x})$ is the initial tracer density at time $t_{0}$, and $\rho\left(t, t_{0}, \mathbf{x}\right)=\rho_{0}\left(\phi_{t}^{t_{0}}(\mathbf{x})\right)$ the tracer density at time $t$, then the variance $\Sigma_{\rho}\left(t, t_{0}\right)$ of $\rho$ should decay exponentially over time and thus can be modeled by

$\Sigma_{\rho}\left(t, t_{0}\right)=A_{0} e^{-r\left|t-t_{0}\right|}+A_{1}$,

The relaxation constant $r$ is called the measured mixing rate (MMR), (Voth et al., 2003). The use of $\left|t-t_{0}\right|$ accounts for both forward and backward time integration, which allows comparison to other Lagrangian methods utilizing forward and backward integration times. It is assumed here that $r$ is representative of the entire system, and the initial tracer profile is not important in a long enough integration time, since the positions of fluid particles in a closed domain eventually become indistinguishable with respect to their initial conditions. For the non-autonomous axisymmetric model, we make this rate space and time dependent by varying the initial spatial region $R$ in which trajectories are seeded, as well as the initial time $t_{0}$.

For $R$ we choose regions in the eye, eyewall updraft, and the boundary layer inflow, which are representative of particular features of the flow. These regions have very different mixing properties, and different associated mixing rates.
Since the fluid in this model does not eventually become homogenized, the mixing rate is a measure of how trajectories characteristic of a certain feature disperse, e.g. become advected through a jet. Trajectories that enter the eyewall updraft exit the domain through the upper level outflow jet, so there are many trajectories that exit the domain in finite time, and there are large regions of the domain that trajectories from the core will not enter. To accomodate this trajectory behavior, a finite-time version of the mixing rate is used here. Trajectories are advected for an integration time such that they remain within the spatial domain. The mixing rate $r\left(R, t_{0}\right)$ then approximates the long time scalar variance decay by the homogenization over a short time. We note that for flows that do not eventually reach a homogenized state, the degree of homogenization, $A_{1} /\left(A_{0}+A_{1}\right)$, can measure how clustered the set remains as it is advected.

\subsection{Finite-time Lyapunov exponents (FTLE's) and La- grangian coherent structures (LCS's)}

Lagrangian coherent structures (LCS's) are finite-time invariant manifolds which are advected by the flow. Particle trajectories do not cross LCS's, but diverge from them exponentially in a direction not aligned with the LCS. LCS's are determined from the rate of maximal expansion of nearby trajectories. A scalar measure of maximal stretching is provided by the field of finite-time Lyapunov exponents (FTLE's) (Shadden et al., 2005). Consider an infinitessimal perturbation $\mathbf{x}_{0}^{\prime}$ of the point $\mathbf{x}_{0}$. After a time $T$, the perturbation becomes

$$
\begin{aligned}
\mathbf{x}^{\prime}\left(t_{0}+T\right) & =\phi_{t_{0}}^{t_{0}+T}\left(\mathbf{x}_{0}+\mathbf{x}_{0}{ }^{\prime}\right)-\phi_{t_{0}}^{t_{0}+T}\left(\mathbf{x}_{0}\right) \\
& =\frac{d \phi_{t_{0}}^{t_{0}+T}\left(\mathbf{x}_{0}\right)}{d \mathbf{x}_{0}} \mathbf{x}_{0}{ }^{\prime}+\mathcal{O}\left(\left\|\mathbf{x}_{0}{ }^{\prime}\right\|^{2}\right) .
\end{aligned}
$$

To find the magnitude of the growth rate of the perturbation, we drop the $\mathcal{O}\left(\left\|\mathbf{x}_{0}^{\prime}\right\|^{2}\right)$ term and take the Euclidean norm

$$
\left\|\mathbf{x}^{\prime}\left(t_{0}+T\right)\right\|=\sqrt{\left\langle\mathbf{x}_{0}^{\prime}, \Delta \mathbf{x}_{0}^{\prime}\right\rangle}
$$

where the matrix

$$
\Delta=\frac{d \phi_{t_{0}}^{t_{0}+T}\left(\mathbf{x}_{0}\right)^{*}}{d \mathbf{x}_{0}} \frac{d \phi_{t_{0}}^{t_{0}+T}\left(\mathbf{x}_{0}\right)}{d \mathbf{x}_{0}}
$$

(the asterisk denotes the transpose of a matrix or vector) is symmetric and gives a finite time representation of the Cauchy-Green deformation tensor. If $\left\|\mathbf{x}_{0}{ }^{\prime}\right\|$ is held constant, the maximal expansion occurs when $\mathbf{x}_{0}^{\prime}$ is aligned with the eigenvector corresponding to the largest eigenvalue, $\lambda_{\max }(\Delta)$, of $\Delta$,

$$
\begin{aligned}
\max _{\mathbf{x}_{0}^{\prime}}\left\|\mathbf{x}^{\prime}\left(t_{0}+T\right)\right\| & =\sqrt{\lambda \max (\Delta)}\left\|\mathbf{x}_{0}^{\prime}\right\| \\
& =\exp \left(\sigma_{t_{0}}^{t_{0}+T}\left(\mathbf{x}_{0}^{\prime}\right)|T|\right)\left\|\mathbf{x}_{0}^{\prime}\right\|,
\end{aligned}
$$


where

$\sigma_{t_{0}}^{t_{0}+T}\left(\mathbf{x}_{0}\right)=\frac{1}{2|T|} \log \lambda_{\max }(\Delta)$

is the largest finite time Lyapunov exponent for the integration time $T$ at the point $\mathbf{x}_{0}$ at initial time $t_{0}$. The FTLE is computed forward $(T>0)$ and backward $(T<0)$ in time, which allows detection of forward time repelling and attracting material lines, respectively.

Lagrangian coherent structures (LCS's) are structures which maintain some particular property when advected with the flow. An initial grid of seeded trajectories can be advected to produce a scalar field of Lagrangian values dependent on initial-time. Since high FTLE values correspond to large separation of trajectories, ridges of FTLE fields have been defined as LCS's corresponding to invariant manifolds by Shadden et al. (2005). The extraction of ridges from a time varying FTLE field is impractical, but the structures are often obvious from visual inspection.

While the exact extraction of LCS's is generally not possible, FTLE's still give the total separation of trajectories within a region, and the statistical distribution of FTLE values allows definition of global mixing rates. Antonsen Jr. et al. (1996) have shown that for autonomous or time-periodic velocity fields in closed and bounded domains, the variance of a tracer coincides with the quantity

$G\left(t, t_{0}\right)=\int \sigma^{(1 / 2)} e^{-\sigma t} P\left(\sigma, t, t_{0}\right) d \sigma$,

where $P\left(\sigma, t, t_{0}\right)$ is the probability distribution function of the FTLE values. For non-steady velocity fields in open domains this coincidence cannot be expected, but by fixing $t_{0}$, the function $G(t)$ may show a similar exponential decay like the tracer variance. Thus, assuming that $G(t)$ has the form

$G(t)=A_{0}^{\prime} e^{-r^{\prime}\left|t-t_{0}\right|}+A_{1}^{\prime}$,

we can solve numerically for $r^{\prime}$ to obtain a predicted FTLE mixing rate (FMR), (Antonsen Jr. et al., 1996). This rate is meant to measure the advective mixing processes determined by initial trajectory separation, and does not account for the diffusive processes that govern the long time mixing. However, the integration time, $T$, used must be sufficiently long so that the FTLE's resolve LCS's, and performing integrations in a moving time frame within a finite time range imposes an upper bound on $T$. The optimal integration time will be discussed in more detail in Sect. 5 .

The FMR method was originally designed for closed bounded domains and steady or time-periodic velocity fields, see (Voth et al., 2003) for an application to a time-periodic velocity field with chaotic trjectories. Since the axisymmetric model has general time dependence, and important mixing properties are localized in time and space, we adapt this measure to include initial time dependence and initial space dependence. The resulting time series of mixing rates are then compared to the time series of the measured mixing rates, and to measures of intensity.

\subsection{Relative dispersion (RD)}

Relative dispersion is based on the average displacement of an ensemble of initially proximate trajectories from a mean particle position, Huber et al. (2001). When an ensemble is taken to be a well defined set of trajectories, relative dispersion can differentiate between sets of initial conditions that have different mixing properties. For a set $R$ with an ensemble of initial conditions $\mathbf{x}_{0} \in R$, the root mean squared (RMS) displacement of the ensemble of trajectories seeded at time $t_{0}$ in $R$ is defined as

$\sigma(t)=\left\langle\|\mathbf{x}(t)-\overline{\mathbf{x}}(t)\|^{2}\right\rangle^{\frac{1}{2}}$

where $\langle$.$\rangle denotes the average over the set, and \overline{\mathbf{x}}$ is the mean particle position. The relative dispersion $K(t)$ is defined by

$K(t)=\frac{1}{2} \frac{d}{d t} \sigma^{2}(t)$,

and $\sigma(t)$ shows a power law relationship for $t \rightarrow t_{0}$ due to the presence of initial velocity correlations, (Huber et al., 2001),

$\sigma(t) \propto\left|t-t_{0}\right|^{\gamma}$

\subsection{Relative dispersion from FTLE's (FRD)}

While FTLE's and relative dispersion are similar measures of trajectory separation, the FMR is not directly comparable to the RD in their given forms. To allow a comparable mixing rate, we define the RMS displacement of an ensemble of trajectories in the direction of maximal expansion through the FTLE values by

$$
\begin{aligned}
D_{t_{0}}^{t}(R) & =\left\langle\left.\exp \left(2 \sigma_{t_{0}}^{t}(\mathbf{x})\left|t-t_{0}\right|\right)\right|^{1 / 2}\right. \\
& \propto\left|t-t_{0}\right|^{\gamma^{\prime}}
\end{aligned}
$$

which gives a power $\gamma^{\prime}$ for $t \rightarrow t_{0}$. The FTLE based relative dispersion (FRD) is then defined by

$K_{f}(t)=\frac{1}{2} \frac{d}{d t} D^{2}(t)$,

and is, for the integration time $T=t-t_{0}$, considered as a function of the region $R$ and $t_{0}$ The FRD can be considered as an average stretching factor for an ensemble of trajectories. In this paper we will use the FRD as a measure of relative dispersion.

\section{Model overview}

The axisymmetric, nonhydrostatic, cloud-resolving hurricane model of Rotunno and Emanuel (1987) is integrated on a staggered $C$ grid using a fixed radial $(3.75 \mathrm{~km})$ and fixed vertical $(312.5 \mathrm{~m})$ grid spacing at one-fourth the originally published grid spacing. Ice physics are not simulated and explicit convection is employed using a fixed precipitation 

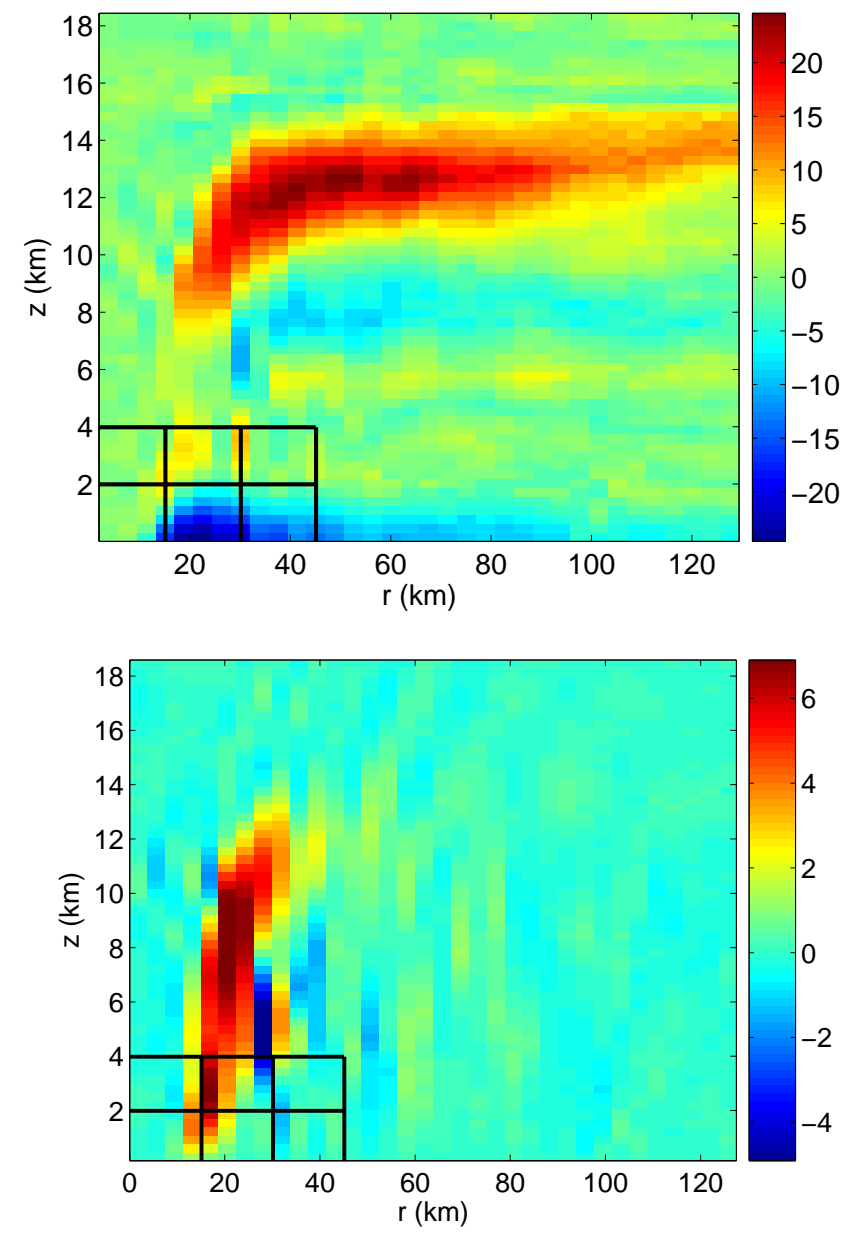

Fig. 1. Radial velocity field (a) and vertical velocity field (b) at $t=400 \mathrm{~min}$. with boxes used for computing mixing rates in the lower left of each image.

fall speed of $7 \mathrm{~ms}^{-1}$. Subgrid-scale turbulence is parameterized using a modified Smagorinsky (1963) formulation with horizontal mixing length of $750 \mathrm{~m}$. Radiation is simply represented by Newtonian relaxation to the initial basic state potential temperature profile with a cooling rate capped at $2 \mathrm{~K} \mathrm{day}^{-1}$. A sponge layer is provided above the model tropopause. Surface fluxes of momentum and enthalpy are conducted with a bulk aerodynamic formulation with the ratio of drag and enthalpy coefficients set to unity and the drag allowed to vary with wind speed by Deacon's formula, Roll (1965). The initial sounding is that of $4 \times$ run by Persing and Montgomery (2003). Data output is at a two-minute interval starting with a time (day 13) when a quasi-steady intensity $\left(\approx 85 \mathrm{~m} \mathrm{~s}^{-1}\right)$ is reached for the simulation.

The $(u, w)$-flow (in the radial/vertical plane of motion) (shown in Fig. 1 at $t=400 \mathrm{~min}$ ) has several dominant characteristics which are often separated by lines of high shearing. The main feature is the axisymmetric eyewall. It appears

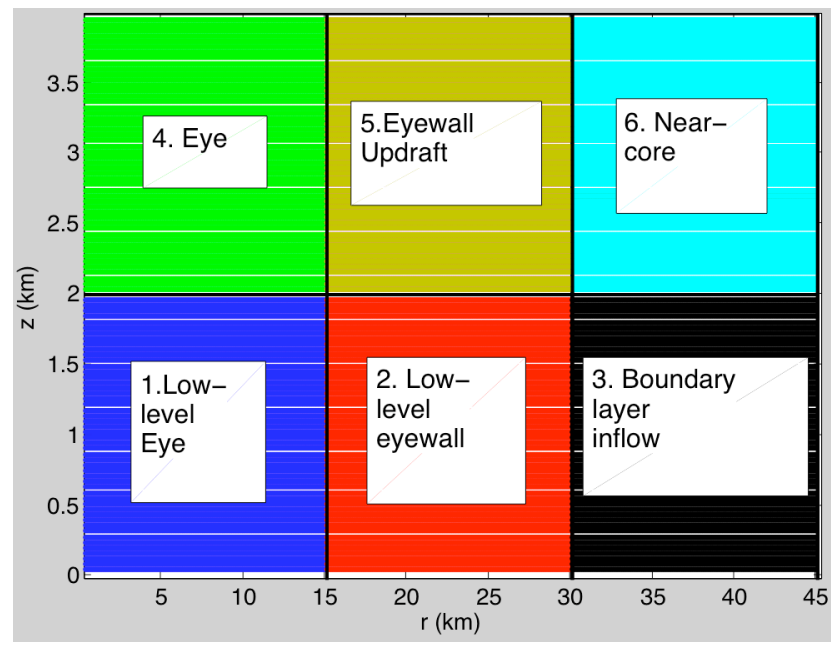

Fig. 2. Locations of boxes in the lower inner core.

as a slanted, vertically oriented structure that separates from the sea surface at approximately $r=20 \mathrm{~km}$, and goes upward to a maximum height of $z=15 \mathrm{~km}$. Inside of the eyewall is the eye which has very slow velocities. The eyewall updraft takes trajectories upward and is separated from the eye by a line of high vertical shearing. The boundary layer inflow is the main source of material that enters into the updraft. As material moves through the updraft, it enters the upper level outflow, where it goes outward and leaves the domain. There is also a midlevel inflow that brings material inward, but with lower inflow velocity than the boundary layer inflow.

\section{Numerical methods}

Mixing rates are computed by adapting the techniques from Sect. 2 to seeded sets of trajectories. Trajectories are advected in radius and height through a fourth order RungeKutta method. Since the locations of seeded particles do not become eventually homogenized throughout the entire domain and some trajectories leave the domain, the initial locations of particles become important.

Trajectories are seeded into initial boxes, which are representative regions for different aspects of the flow (Fig. 2). The boxes are placed in the low level inner core region of the hurricane. The boxes approximately split the eye and eyewall updraft (Fig. 1b), with two boxes in the vertical direction used to distinguish boundary layer properties from other low level properties. The split between the eye and eyewall updraft boxes in the radial direction is placed at the approximate location of the $1 \mathrm{~m} / \mathrm{s}$ vertical wind contour. Two additional boxes are placed outside the eyewall to capture mixing in the boundary layer inflow for the lower box, and convection in the near-core for the upper box. 
Trajectories are seeded in the initial boxes at a resolution of 64 times model resolution in the radial direction and 8 times in the vertical direction, giving 256 by 50 total trajectories for each box. The same resolution of trajectories is used for the computation of the FTLE fields, which gives a balance between clear ridges and computational expense.

The MMR requires the computation of a trajectory density, which is measured for a given box as the ratio of the number of trajectories in the box at a given time divided by the total number of initial trajectories starting in the given seed box. For counting the number of final trajectories, we have divided the entire domain into a 8 vertical by 20 horizontal grid of boxes of the same size as the six representative boxes, allowing trajectory movement into a domain of $16 \mathrm{~km}$ height by $300 \mathrm{~km}$ radius. The variance is then computed from the density in all final boxes.

For a fixed initial time, the mixing rates from the concentration and from FTLE fields both follow an exponential decay as a function of integration time. The mixing rate limit $A_{1}$ is determined by taking the minimum concentration variance over the finite integration time, and the initial value $A_{0}+A_{1}$ is the initial variance. The MMR $r$ is found by taking the $\log$ of the time-series $\Sigma_{\rho}(t)-A_{1}$, where $A_{1}$ is $90 \%$ of the minimum value of $\Sigma_{\rho}(t)$, and the slope of the linear function is found using a linear least-squares best fit. The concentration variance $\Sigma_{\rho}, \log \Sigma_{\rho}\left(t, t_{0}\right)$, and the standard deviation of the error for the best fit over the time interval $\left(t_{0}, t_{0}+T\right)$ are shown in Fig. 3 as functions of $T$.

For the FMR, the initial value $A^{\prime}{ }_{0}+A^{\prime}{ }_{1}$ is determined by the initial FTLE distribution, while the limit $A^{\prime}{ }_{1}$ is again determined by taking $90 \%$ of the minimum value of $G(T)$ over the integration times. The mixing rate $r^{\prime}$ is determined in the same manner as the MMR (Fig. 4).

The FRD is computed from the FTLE values in the six initial boxes to compute an effective RMS displacement. By varying initial time, time-series of mixing rates can be compared to time-series of maximum winds (shown below). The integration time is also varied to view the short and long time aspects of the dispersion. Initial time and integration time are measured in minutes while all distances are in $\mathrm{km}$.

\section{Lagrangian fields}

The Lagrangian fields were calculated for a variety of integration times to capture short and long time mixing processes. Lines of high FTLE values in both the forward and backward time fields mark a transition region between the eye and eyewall (Figs. 5, 6). The FTLE's do not distinguish well between stretching and shear because they are computed over a finite time, and concern only the maximal deformation of a material area element. Shear in particular is associated with the growth of line elements which are initially aligned orthogonal to trajectories, while hyperbolicity pertains to the growth orthogonal to trajectories of line elements aligned or-

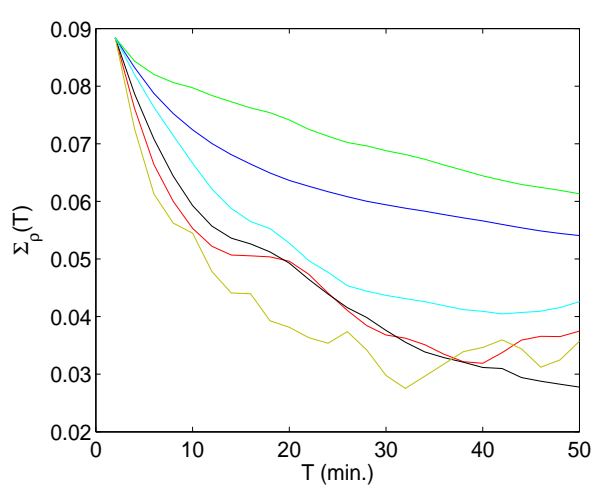

(a)

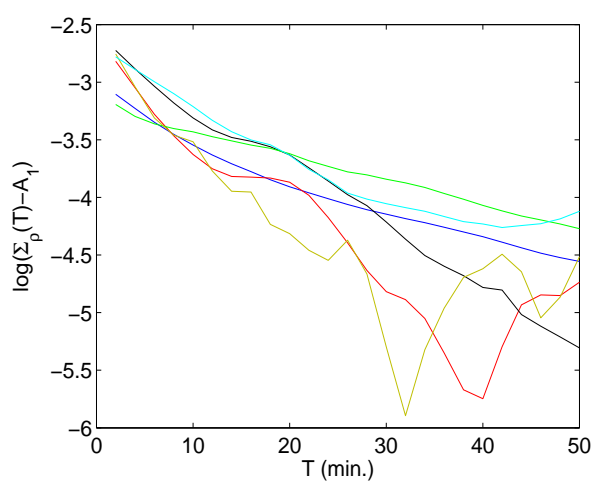

(b)

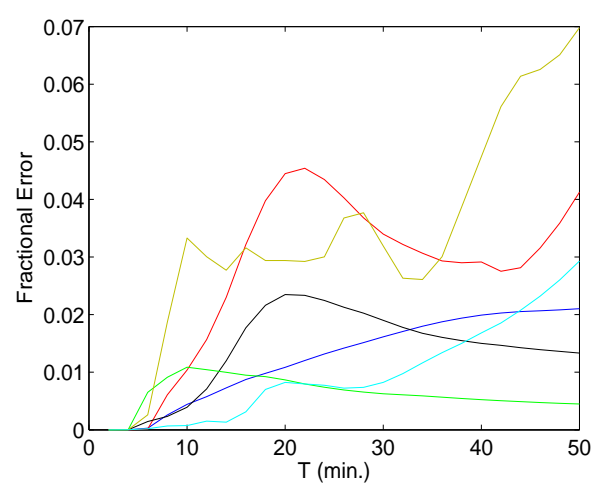

(c)

Fig. 3. Concentration variance $\Sigma_{\rho}\left(t, t_{0}\right)$ (a), $\log \left(\Sigma_{\rho}\left(t, t_{0}\right)-A_{1}\right)$ (b), and relative error (c) plotted versus integration time $T$ for the 6 mixing boxes with trajectories seeded at $t_{0}=400 \mathrm{~min}$. Other initial times give similar decay structure but different quantitative details.

thogonal to the trajectory motion. Trajectories originating in the eyewall updraft reach a radius of $140 \mathrm{~km}$ in the outflow in $120 \mathrm{~min}$, where the outflow jet ${ }^{1}$ governs the mixing, and the low level effects cannot be seen.

\footnotetext{
${ }^{1}$ The cores of jets show low mixing regions (blue) in the FTLE field as there is very little relative advection of neighboring trajectories there. Jets are bounded by high shear regions, which lead to large relative advection (red) and large FTLE values.
} 


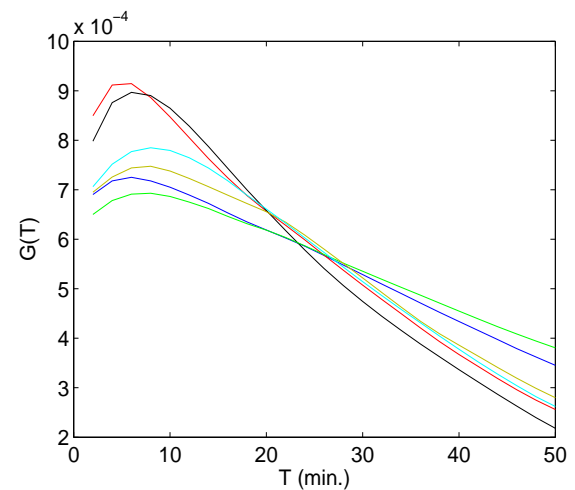

(a)

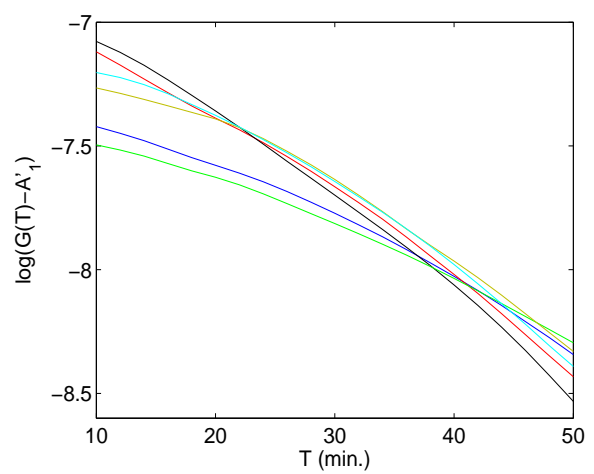

(b)

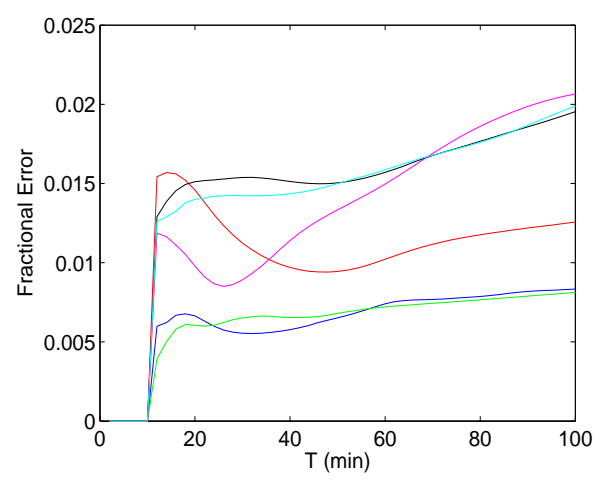

(c)

Fig. 4. FTLE distrbution function $G\left(t, t_{0}\right)(\mathbf{a}), \log \left(G\left(t, t_{0}\right)-A 1^{\prime}\right)$ (b), and relative error (c) plotted versus integration time $T$ for the 6 mixing boxes with trajectories seeded at $t_{0}=400 \mathrm{~min}$. Other initial times give similar decay structure but different quantitative details.

There are several factors to consider in the choice of integration time. The integration time must be chosen long enough so that the LCS's are resolved, and so that the decay functions $\Sigma_{\rho}\left(t_{0}, T\right)$ and $G\left(t_{0}, T\right)$ begin to show an exponential decay. Since the methods aim to capture advective mixing properties, integration time must be chosen short enough so that diffusion is not dominant. Diffusion dominates advection over long integration times, as manifolds lengthen and become indistinguishable.

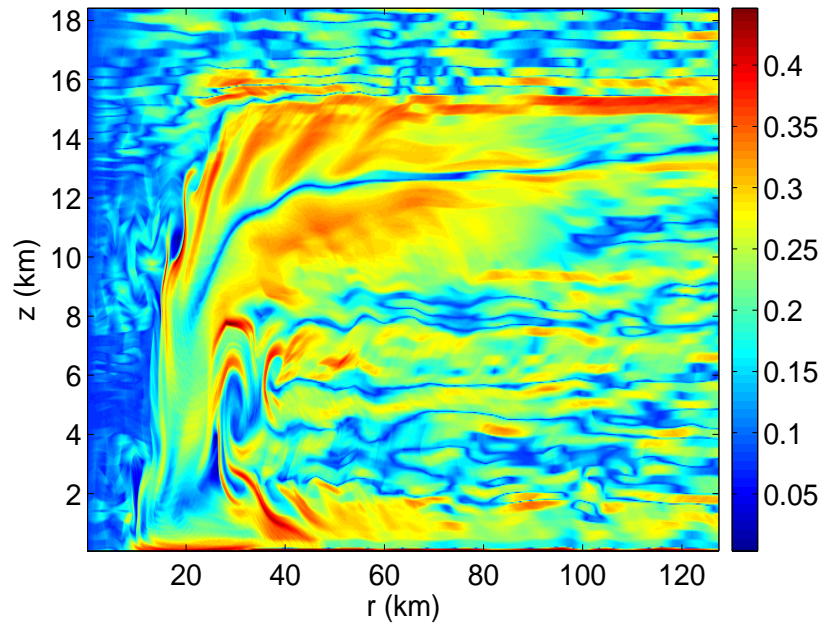

(a)

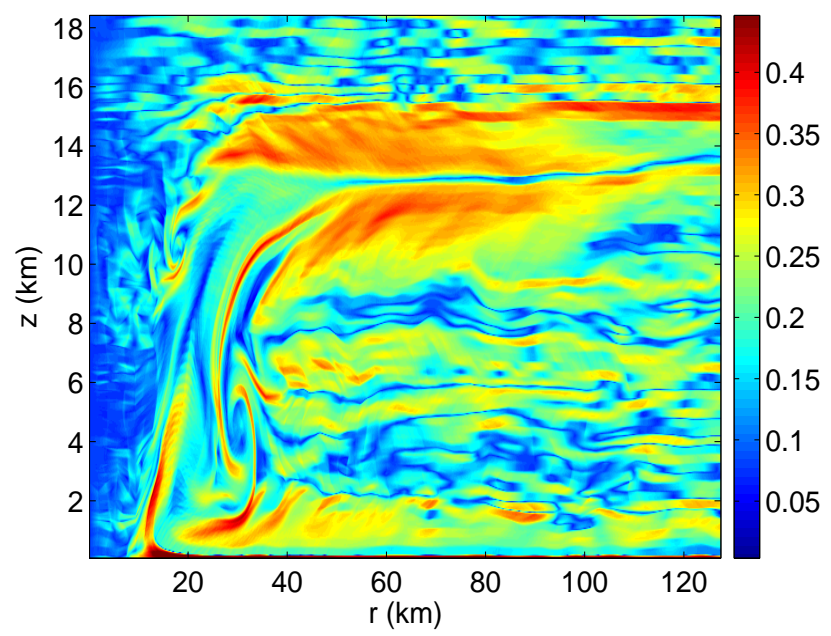

(b)

Fig. 5. Forward (a) and backward (b) time FTLE fields integrated 20 min with an initial time of $400 \mathrm{~min}$.

In addition, the strong time dependence of the velocity field causes the wrapping of manifolds into a tangle, which makes the identification of contigous manifold segments more difficult, and requires a more dense trajectory seeding. Thus a longer integration time imposes computational limitations by requiring more (and longer) trajectory computations.

Dominant flow features such as inflow, outflow and updraft jets govern much of the trajectory movement. High separation often occurs when nearby trajectories split and one enters a jet. LCS's that are strongly hyperbolic cannot exist near a dominant jet, making shearing LCS's vital for characterizing mixing. Fast trajectory flights enable shearing LCS's to be resolved more quickly than hyperbolic LCS's, promoting a shorter integration time. 


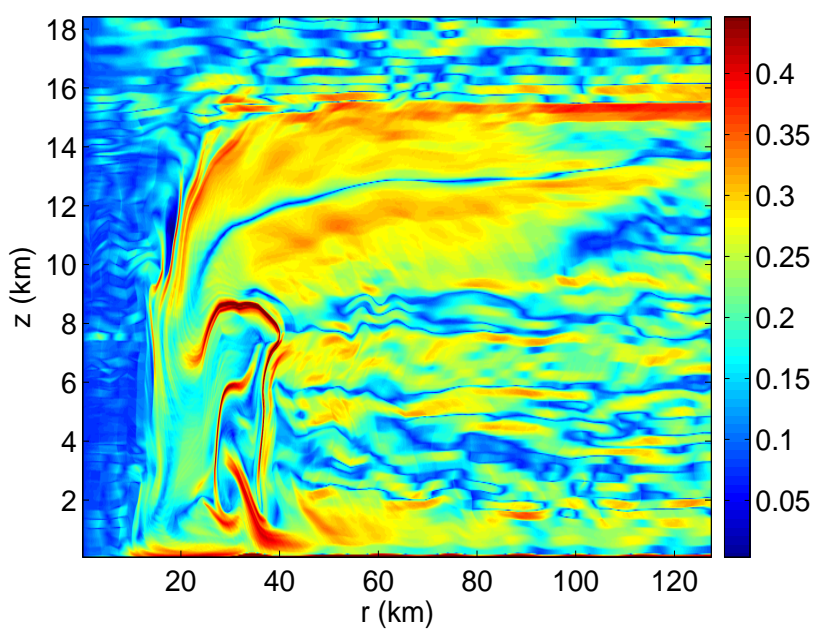

(a)

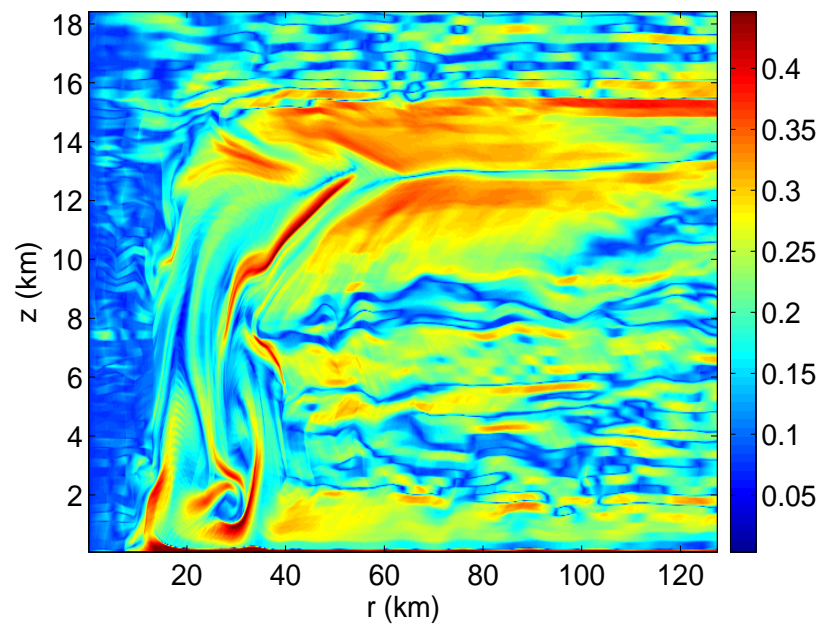

(b)

Fig. 6. Forward (a) and backward (b) time FTLE fields integrated $20 \mathrm{~min}$ with an initial time of $420 \mathrm{~min}$.

\section{Eye-eyewall interaction}

An Eulerian eye-eyewall boundary at low z-levels may be defined as the spatial location that separates the strong upward motion of the eyewall from the weak vertical motion of the eye, e.g. a representative contour of the vertical velocity field, however the strong variation of the velocity field in space and time make such a structure discontinuous in time. From a Lagrangian point of view, the eye-eyewall boundary at low $z$-levels can naturally be defined as the place where there is greatest separation of trajectories, with neighboring trajectories residing in the slow velocity region of the eye and the fast velocity region of the eyewall. This boundary is revealed as a distinguished LCS in the backward FTLE field that persists over all initial times and for integration times

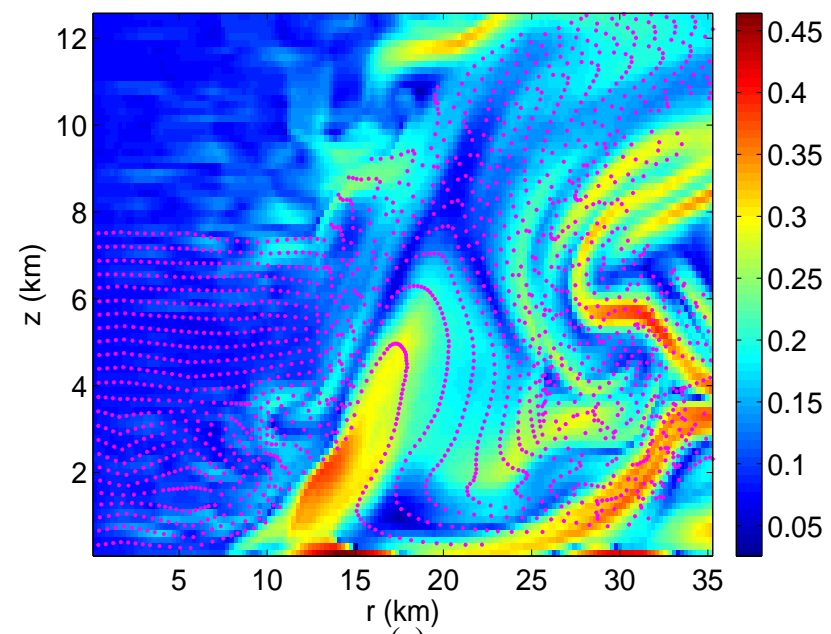

(a)

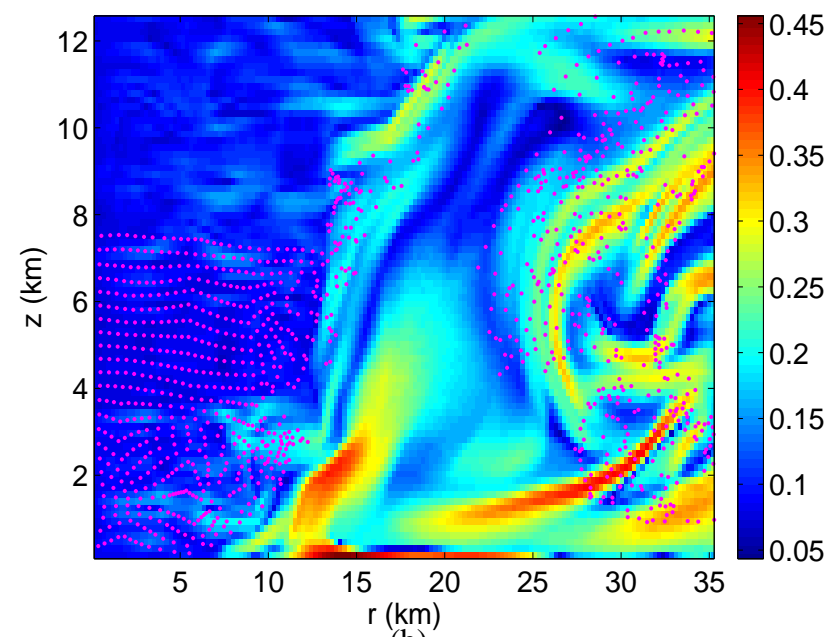

(b)

Fig. 7. Backward time FTLE field integrated $T=-20 \mathrm{~min}$. for initial times of $420 \mathrm{~min}$ (a) and $460 \mathrm{~min}$ (b). The red dots mark the positions at time $t=t_{0}$ of trajectories seeded on a uniform grid at $400 \mathrm{~min}$.

of 20 min. and above, see Fig. 7, 8 (the LCS is marked in Fig. $8 b)$.

The LCS aligns upward from the sea-surface at about $r=15 \mathrm{~km}$ and extends vertically to a height of about $4 \mathrm{~km}$, with nearly the same slope radially outwards for all initial times. It is aligned horizontally and located slightly above the sea-surface for $r>15 \mathrm{~km}$. Although it is found from only a $20 \mathrm{~min}$ integration time, it is invariant for much longer times, and persists over the complete period of analysis of the quasi-steady state intensity. In Fig. $7 \mathrm{a}$ and $\mathrm{b}$, the positions of trajectories seeded on a uniform grid at $400 \mathrm{~min}$ are displayed after 20 and $60 \mathrm{~min}$, superimposed on the backward FTLE fields at initial times of $t_{0}=420$ and $460 \mathrm{~min}$, respectively. The figures show that trajectories in the eye 


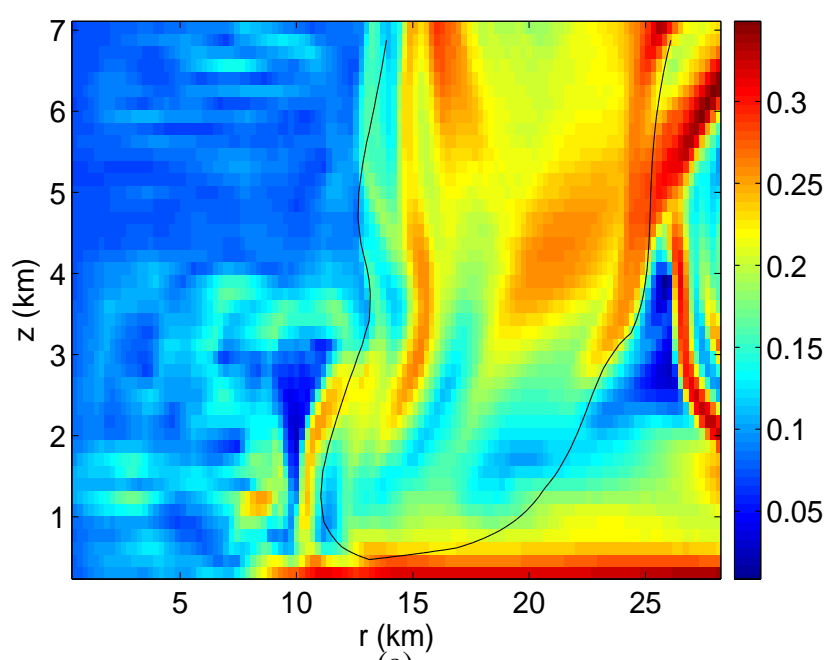

(a)

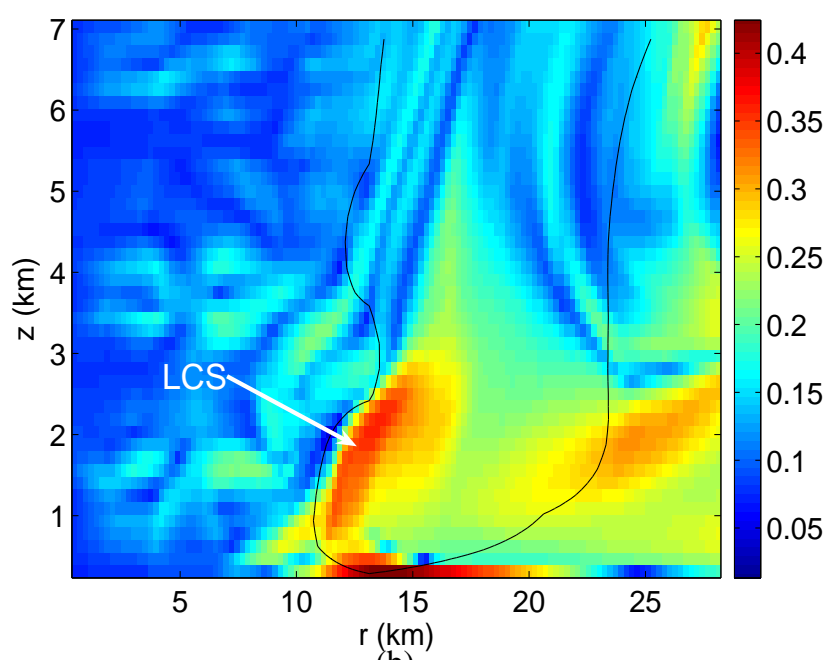

(b)

Fig. 8. Forward (a) and backward (b) time FTLE fields at $t_{0}=$ $400 \mathrm{~min}$ with $1 \mathrm{~m} / \mathrm{s}$ vertical wind contour. The persistent LCS is marked in (b).

have little movement relative to the LCS, while updraft trajectories show strong movement transverse to the LCS. This transverse motion makes the ridge Lagrangian with nearby trajectories quickly leaving it.

In Fig. 8 we show both the forward and backward FTLE fields at $t_{0}=400 \mathrm{~min}$, with the $1 \mathrm{~m} / \mathrm{s}$ Eulerian vertical wind contour superimposed, which may be considered as an Eulerian eye-eyewall boundary. At this time the wind contour is aligned along the LCS; at other times (not shown) it is aligned across the LCS. Seeding trajectories along the LCS (not shown) reveals that trajectories travel transverse to the LCS without crossing, but may cross the Eulerian wind contour.

\section{Steady state approximations}

The velocity fields reside in a quasi-steady state for a period of several hours, between about $400-800 \mathrm{~min}$. During this time period of $400 \mathrm{~min}$ the maximum tangential winds remain in a range of 75 to $88 \mathrm{~m} / \mathrm{s}$, where they oscillate rapidly, but in an aperiodic nature. Aside from the differences in intensity, the velocity fields show structural differences in the periods of high maximum tangential winds and lower maximum tangential winds.

The model gives strong time dependent velocity fields, even in a relatively steady state of intensity, leading to different structures for different initial times. The presence of a secondary convective region outside of the main updraft changes the structures associated with the updraft, and is important for changes in the eyewall near-core flux.

In atmospheric studies, time independent velocity fields are often used to approximate time-dependent flows if the time dependence is small enough that trajectories approximately follow velocity streamlines, see e.g. Dunkerton et al. (2009). Although the time-dependence of this model is too high to infer Lagrangian behavior from Eulerian stagnation points, we make Eulerian approximations associated with high and low wind speeds, and relate them to shorttime effects. We compute two composite-averaged velocity fields from the quasi-steady state period, refered to as strong and weak composites (Fig. 9), which are representative of the phases with strong and weak maximum tangential winds, respectively. The strong (weak) composite is computed by averaging over the instantaneous velocity fields that generate maximum tangential winds at the highest $20 \%$ (lowest 20\%) of maximum tangential winds for the entire time interval of 400-800 min. The vertical component of the strong composite shows a single strong updraft, while the vertical wind field of the weak composite shows a dual updraft structure, but with weaker updraft velocities. The radial velocity fields show a more defined outflow jet for the strong composite.

The composite fields can be considered as time independent velocity fields. The forward and backward FTLE ridges for a time independent velocity field correspond to finitetime, and thus shorter in length, versions of the unstable and stable manifolds of hyperbolic fixed points. Since the velocity field maintains high or low velocities for only a few minutes, invariant structures can only be resolved over a similarly short integration time, and can be viewed as markers for finite-time coherent structures of the full velocity fields. The composite velocity fields yield very different Lagrangian structures, specifically at low levels outside of the updraft, which may affect the path of particles entering the updraft from the BL inflow. The outflow jet takes a straighter path in the strong composite FTLE field. At the boundary layer, the weak composite FTLE field shows high values where the boundary layer inflow meets the eyewall updraft, allowing fewer trajectories to enter the eyewall. The secondary updraft at $35 \mathrm{~km}$ takes some of the boundary layer trajectories 

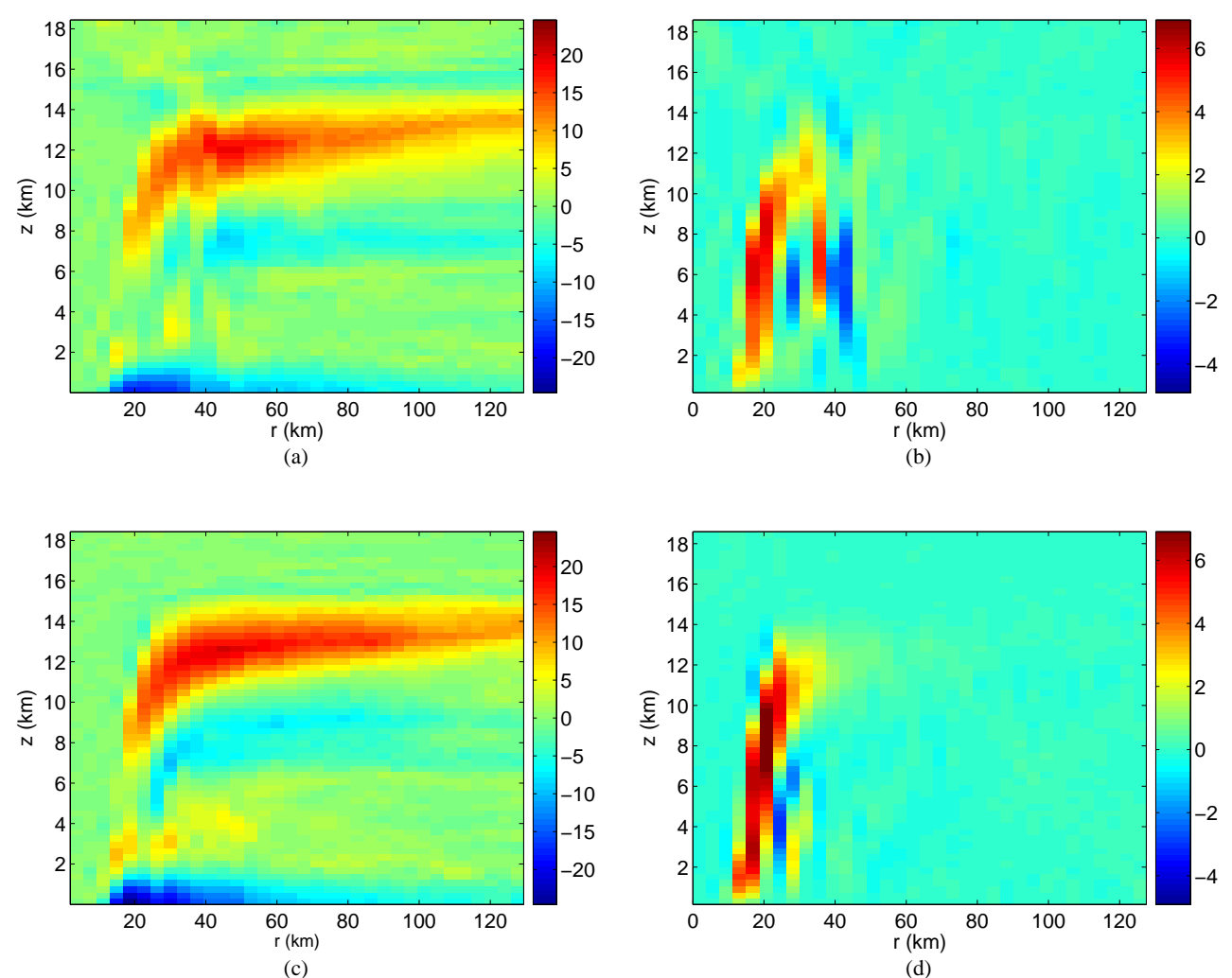

(d)

Fig. 9. Weak composite radial (a) and vertical (b) velocity fields, and strong composite radial (c) and vertical (d) velocity fields.

through a region with several LCS's in the forward and backward FTLE fields of both composite fields (Fig. 10), and moves them upward into the region just outside of the eyewall, before a downdraft takes them inward to the eyewall updraft.

Mixing rates for the composite fields are generally higher for the strong composite field, for both forward and backward integrations, showing that higher intensity coincides with greater mixing. The FRD shows higher values in the boundary-layer inflow region due to the presence of a series of LCS's that cause trajectories to be transported into the eyewall updraft, or recirculation within an eddy that forms during low velocity times in the near-core region.

\section{Time series analysis}

The dependence of the mixing rates on the initial time gives time series that can be analyzed to establish correlations between different quantities. The MMR, FMR and FRD were all computed for different integration times for the sequence of initial times.

For a quasi steady-state hurricane, the connection between intensity and mixing rates is not obvious, especially when the rates are determined by an integration time that lasts longer than a complete period from high to low maximum winds, where the mixing rate value is assigned to the initial time of integration.

The FMR is fit to an exponential decay function, but the curve of the FTLE distribution function does not show a decay for $t-t_{0} \leq 10 \mathrm{~min}$, Fig. 4a. After $10 \mathrm{~min}$, the FMR can be computed by fitting an exponential decay curve to the remaining data, Fig. 4b. The optimal integration time varies for each box, and for initial times. The mixing functions best fit exponential functions at integration times between 20 and $80 \mathrm{~min}$, see Fig. $3 \mathrm{c}$ for the error made in the best fit of the MMR.

In Voth et al. (2003), the MMR has been compared to the FTLE mixing rates for closed domains in time periodic velocity fields, with the FTLE rate being measured at 10 times higher than the measured mixing rate. By allowing initial trajectories to be dispersed into the domain without exiting the domain, the FMR and MMR can be considered as short-time versions of mixing rates within a closed domain. For short time intervals, different initial conditions can lead to significant differences in the mixing rates, as neighboring boxes can show mixing rates differing by a factor of 10 . However, rates in the same box are mostly within a factor of 2 , and the FMR is not always higher. The differences in the variations of the mixing rates found by Voth et al. (2003) and in our study are likely due to our restriction on integration time and due to the nonclosedness of our domain. 


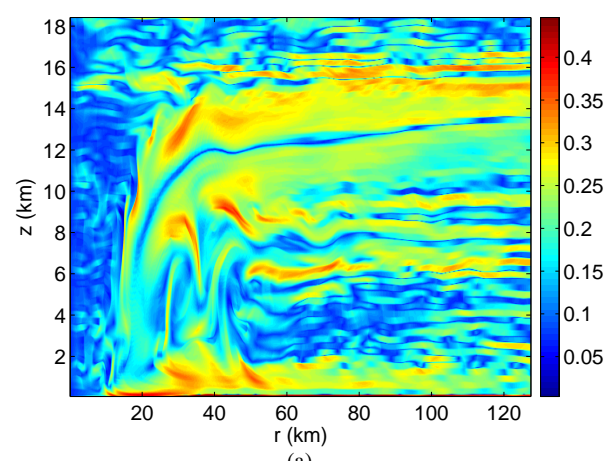

(a)

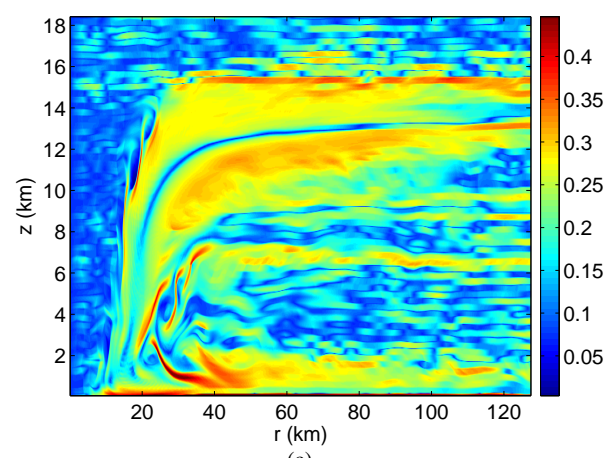

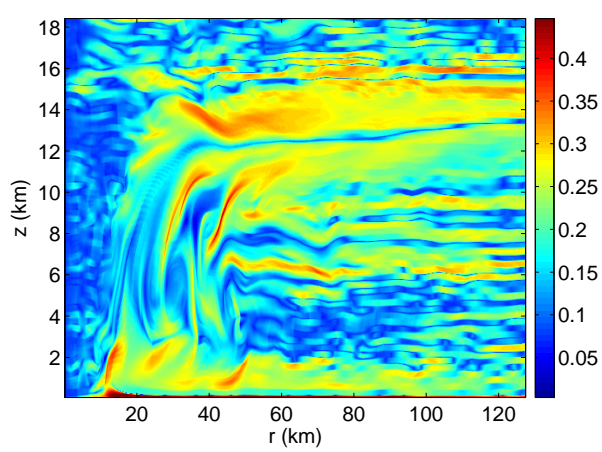

(b)

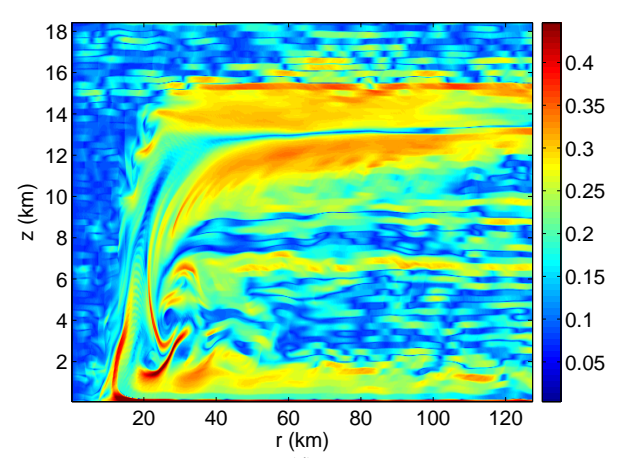

(d)

Fig. 10. Weak composite forward (a) and backward (b) FTLE fields, and strong composite forward (c) and backward (d) FTLE fields, integrated for $20 \mathrm{~min}$.

The FRD, Fig. 11a, with curve fitting error, shown in Fig. $11 \mathrm{~b}$ is fit to a power law (14) for integration times of $20 \mathrm{~min}$ to $100 \mathrm{~min}$. For integration times of $20 \mathrm{~min}$ to $40 \mathrm{~min}$, the RMS displacement, in km, is well fit by the power law, as a portion of the initial set enters the updraft, while other trajectories become temporarily entrained in eddies in the nearcore above the inflow. Trajectories in the near-core region typically reach the outflow in less than $1 \mathrm{~h}$, which provides a reason for a poor power law fit for integration times longer than 1 hour, as trajectories in the outflow show little separation. For longer integration times, an exponential fit may be more appropriate, (Huber et al., 2001), but does not reveal the short-time fluctuations that are characteristic of the time-dependence of this model.

The differences in mixing rates across different boxes for a variety of integration times indicate that the initial boxes do divide the domain into dynamically distinct regions with different mixing properties. In particular, the eye has relatively small trajectory movements compared to the other regions, and all mixing rates are lower in this region. The updraft jet has little separation even with long trajectory flights over short time intervals, and also gives low mixing rates. The highest mixing rates occur at the boundary-layer inflow, where separation from the sea surface and transport of some trajectories into the updraft give a high separation of trajectories.
The time dependent mixing rates can be tested for corrrelation against each of the extrema of the $u, v$, and $w$ winds. All of the mixing rates give higher values when there is higher averaged trajectory separation over a time interval of integration, but the winds are given instantaneously. High particle velocities and velocity gradients at an initial time would indicate high initial separation, but may not correlate to high Lagrangian rates assigned to the same initial time. The structural differences in the strong and weak composite FTLE fields (Fig. 10) indicate that different mixing properties and different structures in the wind fields may coincide with differences in intensity. Correlations of mixing rates to a time lag of maximum winds can indicate the existence of structures which precede or be an effect of higher intensity. Lagrangian structures are an effect of the $(u, w)$-velocity field from previous times in a backward time integration, or future times in a forward time integration. The relation of the Lagrangian structures and mixing rates to instantaneous winds will now be examined.

The maximum tangential winds are taken here as the main indicator of intensity. The azimuthal velocity component is not used for computing trajectories, but is coupled to the radial and vertical velocity component through a system of PDE's. The tangential wind is not periodic, but oscillates between relatively high and relatively low values. 


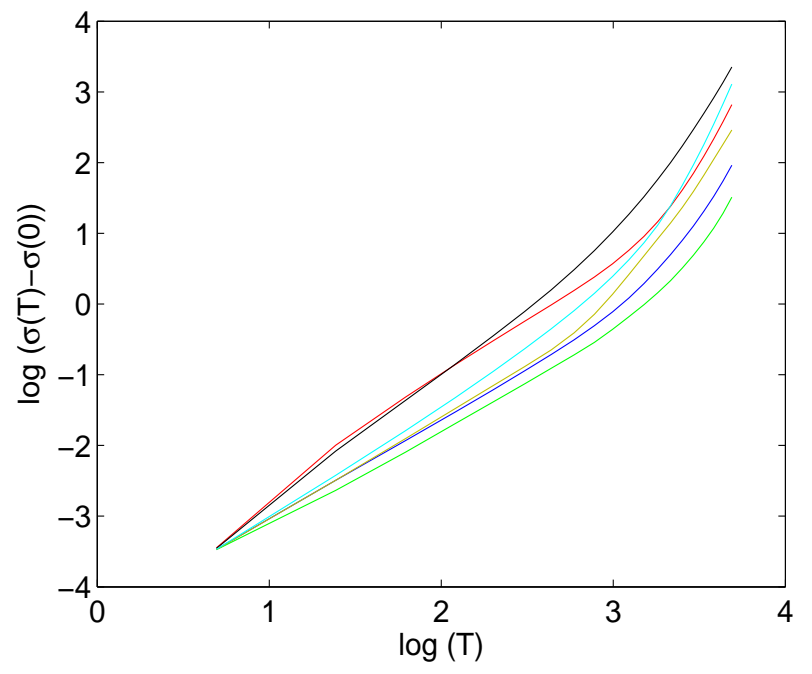

(a)

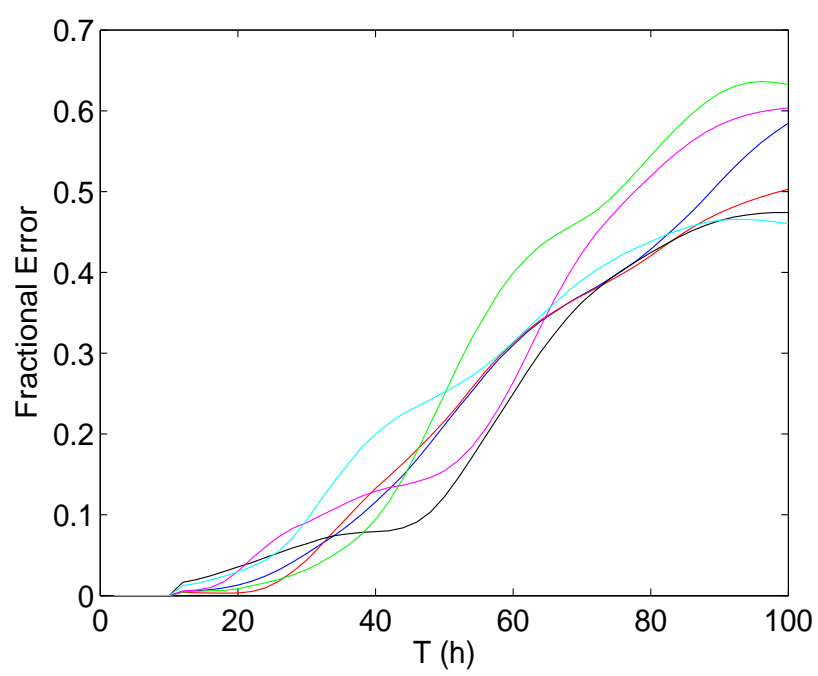

(b)

Fig. 11. Log-log plots of the RMS displacement versus integration time $T$ for FRD (a), and error estimates (b).

Autocorrelation values of maximal tangential winds for time lags above $6 \mathrm{~min}$, computed within the quasi-steady time window, are always below .5 (Fig. 12), showing little predictability within the velocity fields during this time window. Correlations of maximal wind values of the separate velocity components to each other are even less than .2.

The correlations of mixing rates to maximum tangential winds (some correlations above .7) is far greater than to the extrema of radial (correlations below .5) or vertical (correlations below .4) winds.

The oscillations of the maximum tangential winds occur over time intervals of between 20 and $40 \mathrm{~min}$. A $40 \mathrm{~min}$ integration time is below the period of two oscillations, and is

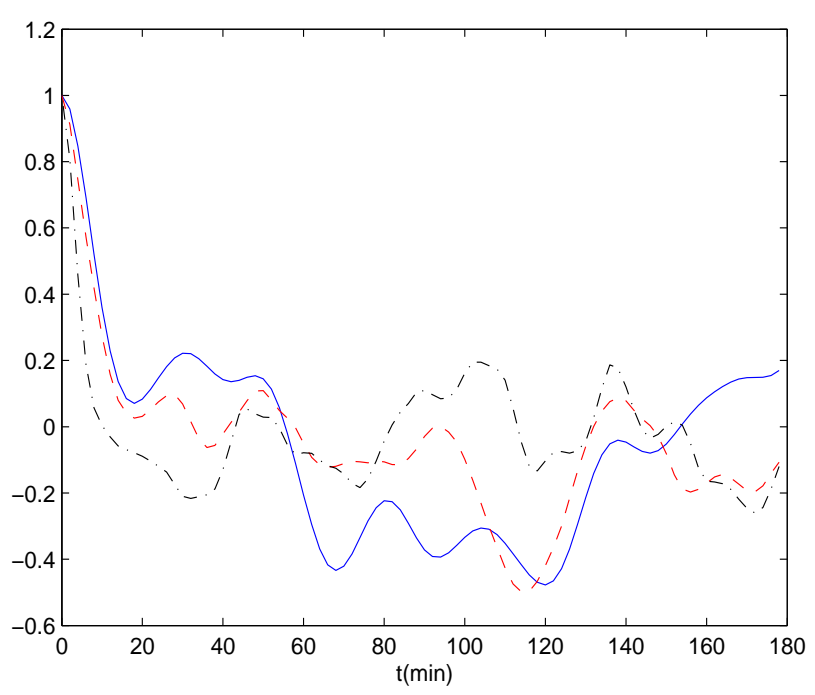

Fig. 12. Autocorrelations of maximal tangential winds (blue), maximal outflow winds (red), and maximal updraft winds (black).

the maximum integration time that yields significant correlation of mixing rates to maximum tangential winds. The rates converge to an exponential or power law after a short time interval, and begin to show correlation after an integration time of $10 \mathrm{~min}$. The best fit to the power law for relative dispersion occurs for integration times of 18 to $40 \mathrm{~min}$, long enough to resolve structures but less than the period of 2 oscillations.

The mixing rates are functions of initial time for each of the six boxes. The different initial boxes give very different mixing rates, with higher mixing rates occuring in the boxes that have the highest velocities. The boundary layer inflow and eyewall updraft boxes show the highest correlations to maximum tangential winds (Figs. 13, 14), for both forward and backward integration time.

Trajectories can be integrated forward or backward in time, giving Lagrangian fields (i.e. MMR, FMR, FRD) that show repelling structures (forward integration), or attracting structures (backward integration). For correlating a Lagrangian quantity to intensity, the forward time integration gives Lagrangian fields that result from future velocities, while backward time integration gives fields that result from past velocities. The wind field at an initial time is predicted by the backward time field at that time, and predicts the forward time field at that initial time. Backward time integration showed higher correlation with the wind fields than forward time integration for most boxes with high correlation.

Correlating the Lagrangian fields to a time lag (Lagrangian fields trailing velocities) or lead (velocities trailing Lagrangian fields) of the velocity field shows how the Lagrangian structures and maximum winds are predictive of each other (Figs. 13, 14). Predicting hurricane intensity (on admittedly very short time scales) by mixing rates can be accomplished by showing a correlation between a backward 


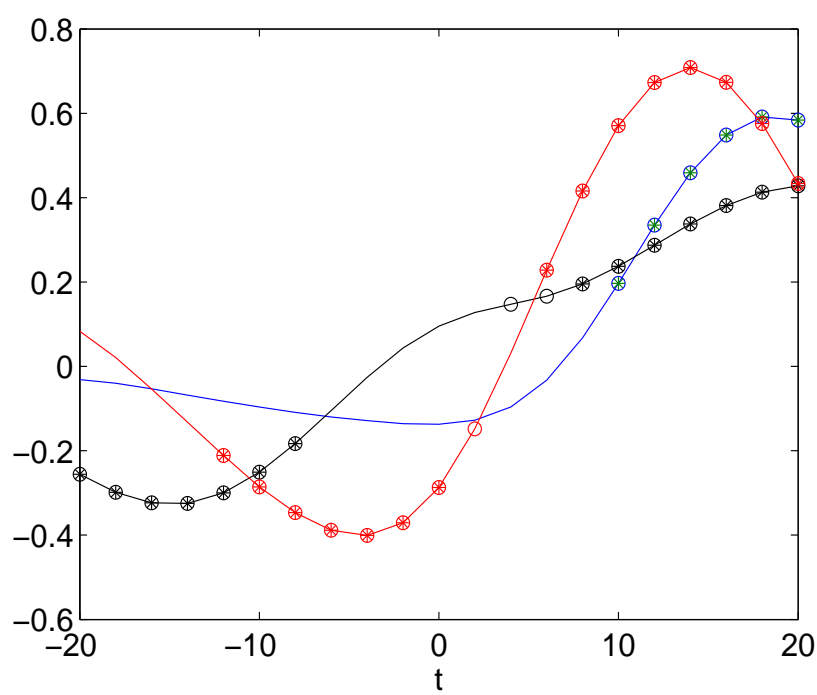

(a)

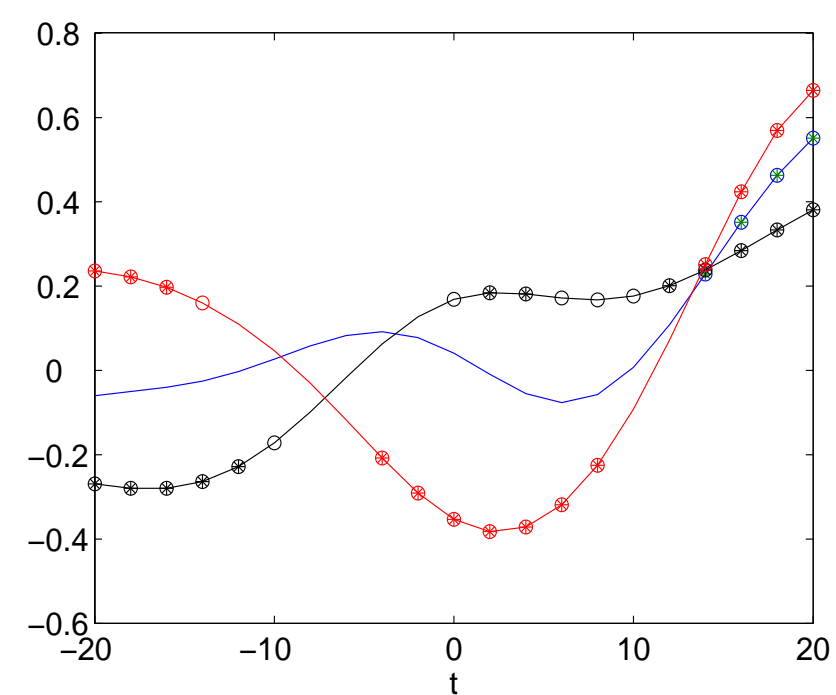

(b)

Fig. 13. Correlations of mixing rates for the BL inflow box lagged by $t$ to maximal tangential winds with (a) $20 \mathrm{~min}$. and (b) $30 \mathrm{~min}$. integration time for FMR (red), FRD (black), and MMR (blue). Filled circles indicate correlation above a $99 \%$ confidence threshold, while open circles indicate correlations above $95 \%$ but below $99 \%$ confidence.

time integration lag, since a function of previous information would correlate to future information.

The FMR shows some correlations to maximal tangential winds above .7, and shows the best correlation of any rate for the BL inflow (Fig. 14(b)). The MMR does not show correlation as high as the other rates, but shows some correlation for the shortest integration time of 20 min (Figs. 14, 15a). The MMR is dependent on the final position of trajectories, and not only on the separation of trajectories. Over longer inte-

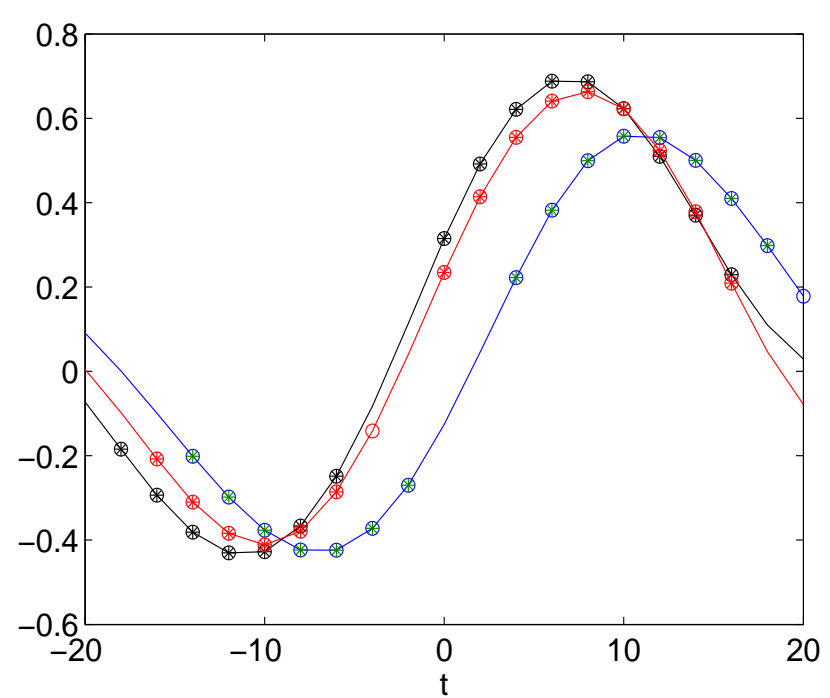

(a)

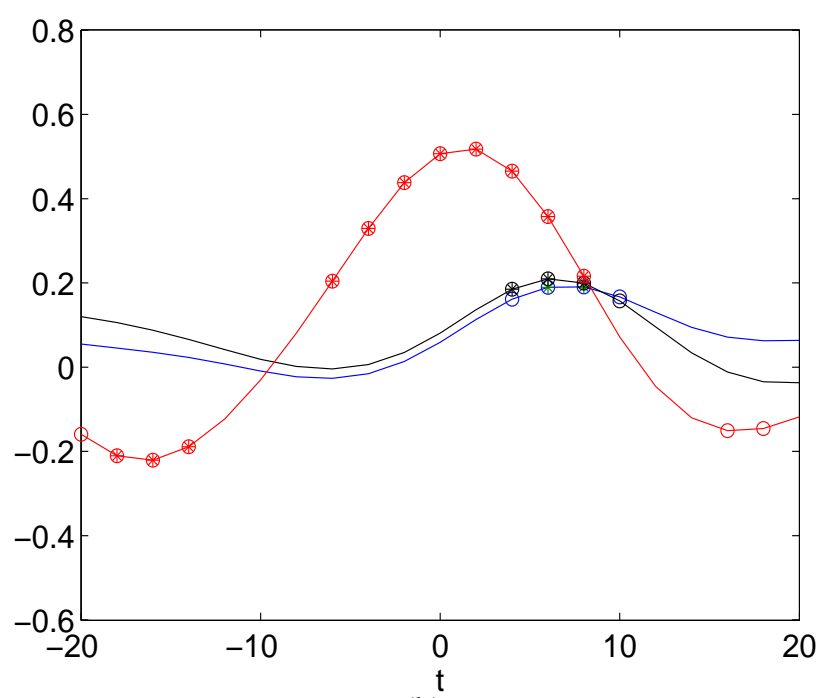

(b)

Fig. 14. Correlations of mixing rates for the BL inflow box (a) and eyewall updraft box (b) lagged by $t$ to maximal tangential winds with $20 \mathrm{~min}$. backward integration time for FMR (red), FRD (black), and MMR (blue). Filled circles indicate correlation above a $99 \%$ confidence threshold, while open circles indicate correlations above $95 \%$ but below $99 \%$ confidence

gration times, this could make the MMR more sensitive to movement caused by gravity waves.

The FRD shows negative correlation of -.6684 to maximal inflow winds (a negative of the extreme minimum of the $u$ field) for the BL inflow box with a forward time integration of $20 \mathrm{~min}$ and a $4 \mathrm{~min}$ time lag, which shows that enhanced mixing is correlated with the enhancement of the BL inflow. The BL inflow has more hyperbolic structures than the other regions, which may make FTLE's better suited as a mixing 


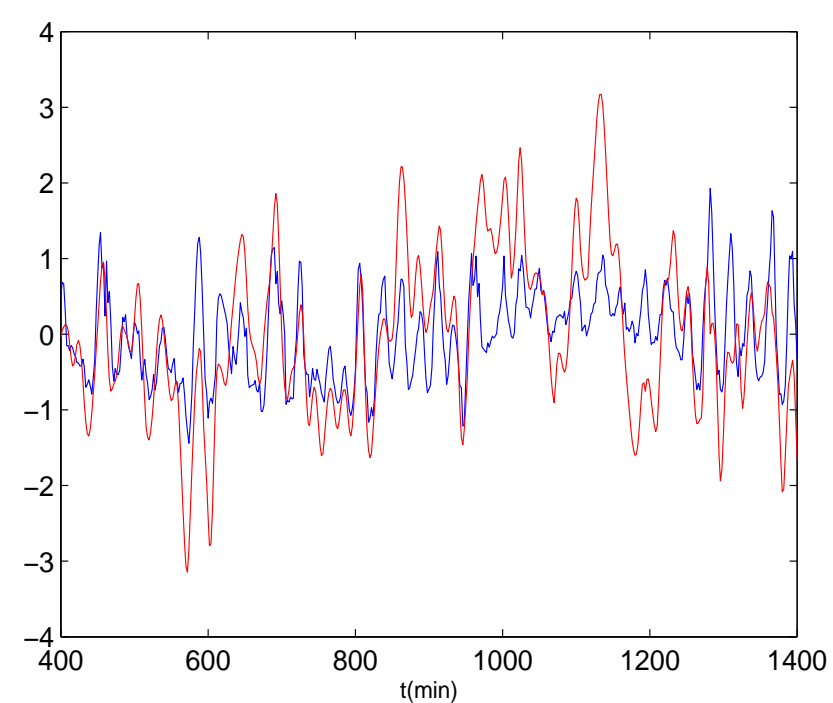

(a)
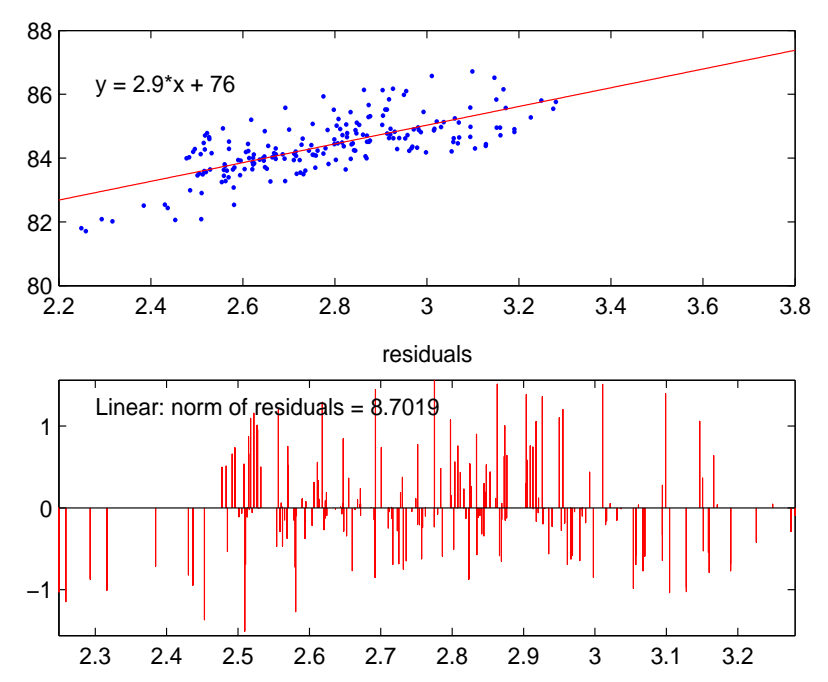

(b)

Fig. 15. (a) Normalized MMR for 20 min backward integration time in eyewall updraaft (blue), and maximum tangential winds delayed $10 \mathrm{~min}$ (red). (b) FRD for $20 \mathrm{~min}$ backward integration time against maximum tangential winds delayed $4 \mathrm{~min}$ for the BL inflow, with linear best fit and norm of residuals.

measure in this region, which can be seen in high correlations for the FRD and FMR (Fig. 13). The eyewall updraft box also shows negative correlation for the FMR to maximal tangential winds with a forward time integration of 20 or 30 min (Fig. 13).

Higher velocities are generally associated with higher mixing rates, which may precede or trail higher intensities. A lead or lag of mixing rates to velocities is then appropriate to capture the effects of mixing. In many cases, the correlation improved when the Lagrangian rates were lagged against the maximum winds. The Lagrangian structures are then predictive of maximum tangential winds. Correlation of 0.6 or higher is present for a lag of up to $10 \mathrm{~min}$, which is about half of a period of oscillation of the maximum tangential winds. The highest correlations occur for 2 to $6 \mathrm{~min}$ lags, which means that the initial time of integration for the Lagrangian fields is at a time where the maximum tangential winds are increasing, but before the local maximum occurs.

\section{Conclusions}

Lagrangian mixing for the complex velocity fields of the axisymmetric hurricane model of Rotunno and Emanuel (1987) has been studied. The inner core region was shown to have Lagrangian structures that vary over time, and play a prominent role for mixing in the region, which is related to hurricane intensity. We have produced mixing rates that correlate to maximum winds, and can be used for a short time prediction of the maximum winds. The mixing rates computed in our study are an extension of mixing rates of Antonsen Jr. et al. (1996) and Huber et al. (2001) established for closed regions or time-periodic velocity fields. In particular, our rates depend on initial time, integration time, time lag, and two spatial coordinates. Various measures of maximal Eulerian intensity have been extracted from the $u, w$, and $v$ wind fields, and compared to the time-dependent mixing rates. A correlation analysis showed that the rates have highest correlation to the maximum tangential winds. The conclusions drawn are that episodes of enhanced mixing between the lowlevel eye and eyewall preceed short-time enhancements of intensity, and thus favor the interpretation that new local generation of buoyancy at the eyewall lead to enhanced thermodynamic cycling of the hurricane heat engine. In principle, the mixing could have been responsive of short-term fluctuations of intensity in response to enhanced flow gradients, or mixing could have directly spun down tangential winds through angular momentum mixing, but since mixing precedes such episodes, neither of these explanations can be favored by the present results. Further work will use a canonical correlation analysis to find correlations between the mixing rates as well as the maximal winds. The methods presented here will also be extended to a three-dimensional hurricane model.

Acknowledgements. This work was supported by NSF Cooperative Agreement ATM-0715426. We thank Wayne Schubert, Eric Hendricks, Arthur Jamshidi, Kate Musgrave, Christopher Rozoff, Jonathan Vigh, and Michael Riemer for their discussions. We also thank the referees for valuable comments that helped to improve the presentation of the paper.

Edited by: P. Haynes 


\section{References}

Antonsen Jr., T. M., Zhencan Fan, Ott, E., and Garcia-Lopez, E.: The role of chaotic orbits in the determination of power spectra of passive scalars, Phys. Fluids, 8, 3094-3104, 1996.

Artale, V., Boffetta, G., Celani, A., Cencini, M., and Vulpiani, A.: Dispersion of passive tracers in closed basins: Beyond the diffusion coefficient, Phys. Fluids, 9, 3162-3171, 1997.

Braun, S. A.: A cloud-resolving simulation of Hurricane Bob (1991): Storm structure and eyewall buoyancy, Mon. Weather Rev., 130, 1573-1592, 2002.

Cram, T. A., Persing, J., Montgomery, M. T., and Braun, S. A.: A Lagrangian trajectory view on transport and mixing processes between the eye, eyewall, and environment using a high resolution simulation of hurricane Bonnie (1998), J. Atmos. Sci., 64, 1835-1856, 2007.

d'Ovidio, F., Fernandez, V., and Hernandez-Garcia, E.: Mixing structures in the Mediterranean Sea from finite-size Lyapunov exponents, Geophy. Res. Lett., 31(17), L17203, doi:10.1029/2004GL020328, 2004.

Dunkerton, T. J., Montgomery, M. T., and Wang, Z.: Tropical cyclogenesis in a tropical wave critical layer: easterly waves, Atmos. Chem. Phys., 9, 5587-5646, doi:10.5194/acp-9-5587-2009, 2009.

Frank, W. M. and Ritchie, E. A.: Effects of environmental flow upon tropical cyclone structure, Mon. Weather Rev., 127, 2044-2061, 1999.

Frank, W. M. and Ritchie, E. A.: Effects of vertical wind shear on the intensity and structure of numerically simulated hurricanes., Mon. Weather Rev., 129, 2249-2269, 2001.

Green, M. A., Rowley, C., and Haller, G.: Detection of Lagrangian coherent structures in 3D turbulence, J. Fluid Mech., 2006.

Haller, G.: Distinguished material surfaces and coherent structures in three-dimensional fluid flows, Physica D, 149, 248-277, 2001.

Haller, G.: Finding finite-time invariant manifolds in twodimensioanl velocity fields, Chaos, 10, 99-108, 2000.

Haller, G.: Lagrangian coherent structures from approximate velocity data, Phys. Fluid., 14, 1851-1861, 2002.

Haller, G.: Exact theory of unsteady separation for two-dimensional flows, J. Fluid Mech., 512, 257-311, 2004.

Haller, G. and Poje, A.: Finite time transport in aperiodic flows, Physica D, 119, 352-380, 1997.

Haller, G. and Yuan, G.: Lagrangian coherent structures and mixing in two-dimensional turbulence, Physica D, 147, 352-370, 2000.

Huber, M., McWilliams, J. C., and Ghil, M.: A climatology of turbulent dispersion in the troposphere, J. Atmos. Sci., 58, 23772394, 2001.

Ide, K., Small, D., and Wiggins, S.: Distinguished hyperbolic trajectories in time-dependent fluid flows: analytical and computational approach for velocity fields defined as data sets, Nonlinear Processes in Geophysics, 9(3/4), 237-263, 2002.

Koh, T. Y. and Legras, B.: Hyperbolic lines and the stratospheric polar vortex, Chaos, 12(2), 382-394, 2002.

Kossin, J. and Eastin, M. D.: Two distinct regimes in the kinematic and thermodynamic structure of the hurricane eyewall, J. Atmos. Sci., 58, 1079-1090, 2001.
Kossin, J. and Schubert, W. H.: Mesovortices, polygonal flow patterns, and rapid pressure falls in hurricane-like vortices, J. Atmos. Sci., 58, 2196-2209, 2001.

Malhotra, N. and Wiggins, S.: Geometric structures, lobe dynamics, and Lagrangian transport in flows with aperiodic timedependence, with applications to Rossby wave flow, J. Nonlinear Sci., 8, 401-456, 1999.

Montgomery, M. T., Bell, M. M., S. A. and Black, M.: Hurricane Isabel (2003): New insights into the physics of intense storms. Part I: Mean vortex structure and maximum intensity estimates., B. Am. Meteorol. Soc., 87, 1349-1354, 2006.

Ottino, J. M.: The kinematics of mixing: stretching, chaos, and transport, Cambridge University Press, New York, USA, 1989.

Persing, J. and Montgomery, M. T.: Hurricane superintensity, J. Atmos. Sci., 60, 2349-2371, 2003.

Smith, R. K., Montgomery, M. T., and Zhu, H.: Buoyancy in tropical cyclones and other rapidly rotating atmospheric vortices, Dynam. Atmos. Oceans, 40, 189-208, 2005.

Roll, H.: Physics of the marine atmosphere, Academic Press, 426 pp., 1965.

Rotunno, R. and Emanuel, K.: An air-sea interaction theory for tropical cyclones. Part II:evolutionary study using a nonhydrostatic axisymmetric numerical model, J. Atmos. Sci., 44, 542561, 1987.

Rutherford, B., Dangelmayr, G., Persing, J., Schubert, W. H., and Montgomery, M. T.: Advective mixing in a nondivergent barotropic hurricane model, Atmos. Chem. Phys., 10, 475-497, doi:10.5194/acp-10-475-2010, 2010.

Salman, H., Ide, K., and Jones, C. K. R. T.: Using flow geometry for drifter deployment in Lagrangian data assimilation, Tellus A., 60(2), 321-335, 2008.

Schubert, W. H., Montgomery, M. T., Taft, R. K., Guinn, T. A, Fulton, S. R., Kossin, J. P., and Edwards, J. P. : Polygonal eyewalls, asymmetric eye contraction, and potential vorticity mixing in hurricanes, J. Atmos. Sci., 56, 1197-1223, 1999.

Shadden, S. C., Lekein, F., and Marsden, J. E.: Definition and properties of Lagrangian coherent structures from finite-time Lyapunov exponents in two-dimensional aperiodic flows, Physica D, 212, 271-304, 2005.

Smagorinsky, J.: General circulation experiments with the primitive equation. I: The basic experiment, Mon. Wea. Rev., 91, 99-164, 1963.

Surana, A. and Haller, G.: Ghost manifolds in slow-fast systems, with applications to unsteady fluid flow separation, Physica D, 237 1507-1529, 2008.

Voth, G. A. and T.C. Saint, G. D., and Gollub, J.: Mixing rates and symmetry breaking in two-dimensional chaotic flow, Phys. Fluid., 15, 2560-2566, 2003.

Willoughby, H. E.: Tropical cyclone eye thermodynamics, Mon. Weather Rev., 126, 3053-3067, 2001.

Zhang, D. L., Y. L. and Yau, M. K.: A multiscale numerical study of Hurricane Andrew (1992). Part V: Inner core thermodynamics, Mon. Weather Rev., 130, 2745-2763, 2002. 